\title{
Build Architecture Design for an Intelligent Security System based on Behavior Tracking
}

\author{
Abdul Monem S. Rahma, Ph.D \\ Computer Science Department \\ University of Technology \\ Baghdad - Iraq
}

\author{
Abeer Salim Jamil, M .Sc \\ Computer Science \& Information Systems \\ Department \\ Al-Mansour University College (MUC) \\ Baghdad- Iraq
}

\begin{abstract}
Tin this paper, we investigate architecture design for the smart environment of an intelligent video security system in an academic environment to achieve a high detection rate with a low false alarm rate for tracking human behavior in video sequences. This intelligent, real-time, and continuous monitoring system can access activity and recognition behavior track across a network of IP cameras.

The system divided into two blocks: The first is the tracking system, which analyzes the movement pattern. The second is the decision system, which can define if the behavior is normal or abnormal and generate alarms. The experimental results for the real-time video streams show the design's effectiveness in recognizing and evaluating human activity.
\end{abstract}

\section{Key words}

Real-time detection, intelligent video security, behavior track detection, IP camera

\section{INTRODUCTION}

Video security has been one of the most active research areas in computer vision. The goal is to efficiently extract useful information from a large amount of video footage collected by IP cameras by automatically detecting, tracking, and recognizing objects of interest, and understanding and analyzing their activities, as shown in Figure 1 [1].

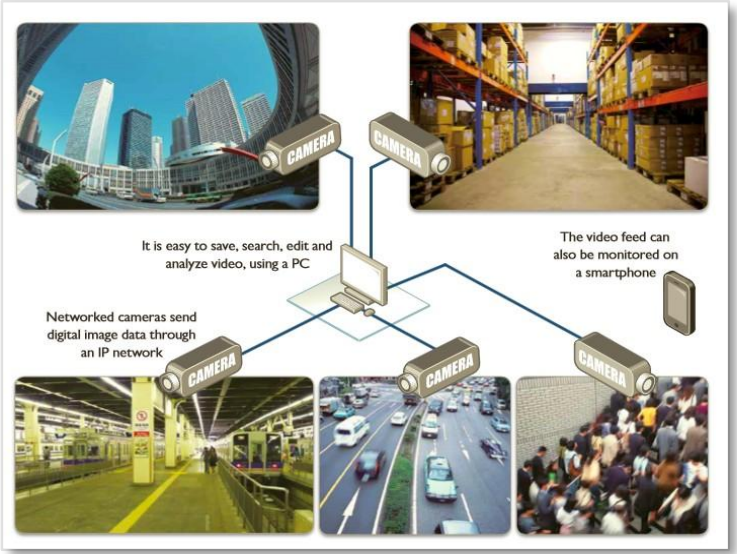

Figure 1: IP cameras View

Video security has a wide variety of applications, in public and private environments, such as homeland security, accident prediction and detection, and patient monitoring. Interest is increasing in video security due to the growing availability of cheap sensors and processors, and the growing need for public safety and security[2].
Researchers are urged to develop intelligent systems to efficiently extract information from large-scale data. To monitor a wide area, such as tracking a vehicle traveling through the road network of a city or analyzing global activities in a large train station, video streams from multiple cameras must be used. Many intelligent multi-camera video surveillance systems have been developed; this multidisciplinary field is related to computer vision, pattern recognition, signal processing, communication, embedded computing, and image sensors [3].

Video security systems constitute a network of video sensors observing people as well as other moving and interacting objects in a given environment for patterns of normal and abnormal activities, interesting events, and other domainspecific goals. However, detecting and tracking objects is harder to address given the requirement that the video security systems must operate in widely varying weather conditions and during all time periods. Computer vision-based solutions have the potential for very discriminating detection and very low false alarm rates. Video security systems have long been used to monitor security-sensitive areas[3].

\section{OBJECTIVE OF VIDEO SECURITY}

The objective of this paper is to describe the development of an intelligent security system for an academic environment. This prototype system incorporates a wide range of advanced security techniques: real-time moving object detection and tracking from stationary camera platforms, specific control and multi-camera cooperative tracking, and human activity recognition and analysis.

\section{ARCHITECTURE DESIGN SYSTEM INTELLIGENT VIDEO SECURITY}

Video security is increasingly found in academic institutions. It is used to oversee the safety of faculty members, staff, and students, as well as to protect assets from vandalism and theft. In this environment, video security is used in particular for monitoring access to the institution's perimeter and detect and follows acts of vandalism and theft.

Since educational institutions often have an IP network infrastructure, setting up digital video security systems is beneficial. Therefore, we implemented our intelligent video system in our university for testing. The system is composed of a set of IP cameras plugged directly into the local network. A human computer interface and a storage space are also plugged intothis system. The main goal is to create an adaptive system that is flexible enough to handle variations in multiple moving objects and other arbitrary changes to the observed scene. 
The architecture enables a single human operator to monitor activities over a broad area using a distributed network of video sensors. The architecture of the system represents the link between image processing and video content analysis as seen in Figure 2.

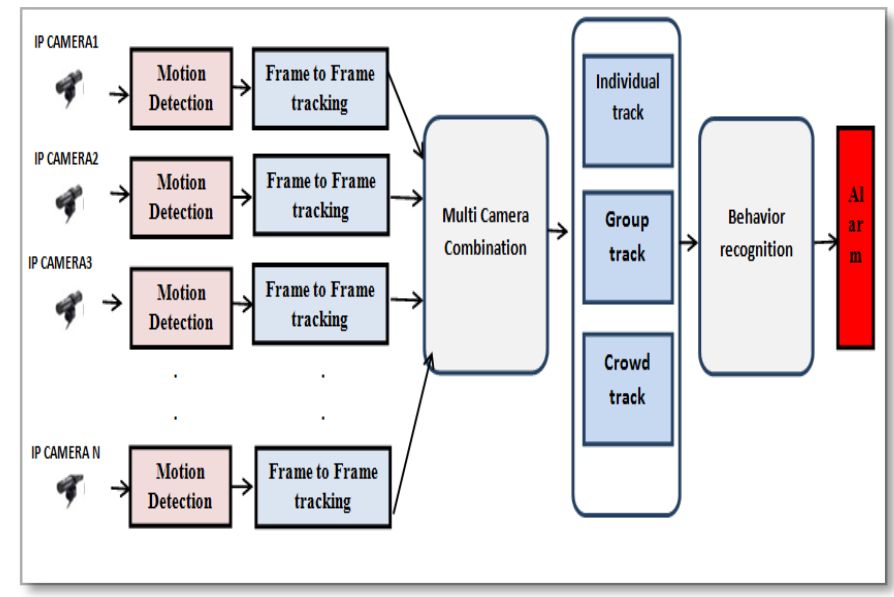

Figure 2: Architecture design for intelligent video Security

This system focuses on monitoring human posture-based events in an education environment with a focus on detecting three types of falls. We considered comprehensive movement scenarios consisting of normal life activities such as walking, running, bending down, sitting down, and lying down, abnormal behaviors such as limping or stumbling, and unusual events such as falling. Since we are interested in analyzing motion occurring during a given window of time, first we need to segment moving objects. Therefore, a background estimation procedure is performed to separate motion from the background. Then features are extracted. Since changes in the human shape can discriminate if the detected motion is normal (e.g., the person walks or sits) or abnormal (e.g., the person falls), we analyzed the shape changes of the extracted shapes in the video sequence.

\section{BEHAVIOR TRACKING DETECTION TECHNIQUES}

\subsection{Motion detection and human tracking[8]}

Video capturing and monitoring devices are used to record video stream, and the frames of the image are extracted. Pattern matching is applied to the sequence of frames with the feature. The background subtraction method is applied to the static background images. Background subtraction compares the reference image and the current image pixel by pixel as shown in the following equation:

$$
\begin{aligned}
& |P(X 1, Y 1) c-P(X 2, Y 2) b k|>T h \\
& \text { Where: }
\end{aligned}
$$

$$
\begin{array}{ll}
P(X 1, Y 1) C & \text { is current frame pixel. } \\
P(X 2, Y 2) b k & \text { is reference frame. } \\
\text { Th } & \text { is threshold value. }
\end{array}
$$

For tracking any object, these features play an important role. Tracking can be categorized into two types: One is region based, and the other is contour based. In region-based tracking, the features of the blob are detected from two consecutive images. If the features match, then these two frame objects are related. In contour-based tracking, the energy of the boundary or contour of the blob is detected from both frames. If the energy of the boundary matches, then the same object is present in both images $[9,10]$.

\subsection{Behavior Recognition [1]}

We have defined four types of methods depending on the type of entity:

i Basic properties methods: We use dedicated routines to compute properties characterizing humans such as trajectory, speed, and direction.

ii State methods: We use numerical methods that include computing:

(a) Distance for states dealing with spatial relations(individual track).

(b) Evolution of temporal features for states dealing with temporal relations (e.g., tracking group).

(c) Speed for states dealing with spatial temporal relations (e.g., "an individual is walking").

iii Event methods: We compare the status of states at two consecutive time points. The output of an event method is Boolean: The event is either detected or not detected. For example ,the event "a group of people enters a zone of interest" is detected when the state "a group of people is inside a zone of interest " changes from false to true.

iv Scenario methods: For simple scenarios (composed of only one state), we verify that a state has been detected during a predefined time period using a temporal filter [2].

\subsection{Activity analysis alarm}

The algorithm stores the information about the detected human after detecting and tracking the object since a human being activity analysis provides intelligence for the process. When an action such as moving into a highly secure area, moving faster than the speed limit, a pose that is not typical, any abnormal activity, or any sign of unusual activity is found, then an alarm is activated[3].

\section{EXPERIMENTAL RESULTS AND DISCUSSION}

\section{1. $\quad$ Experimental setup}

To evaluate the overall system performance, we apply the approach to video footage recorded at our academic institution. Here, we consider indoor environment settings with a single fixed camera motion static scene. The distance from the person to the camera is approximately 3-6 meters. We expected three kinds of behavior: Normal, Unusual, and Abnormal.

a. Normal Activity: Five normal activities are considered: walking, running, bending down to pick something and rising up, sitting down on the floor and standing up, and lying down, as shown in Figure 3. 

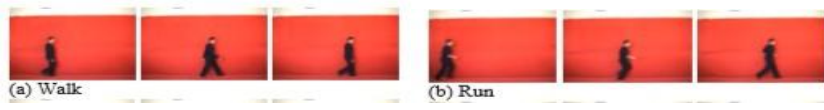

(a) Walking

(b) Running,
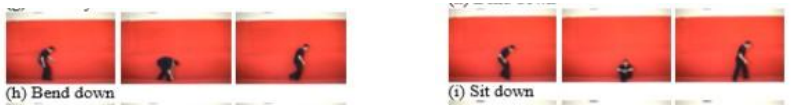

(c) Bending

(d) Sitting down
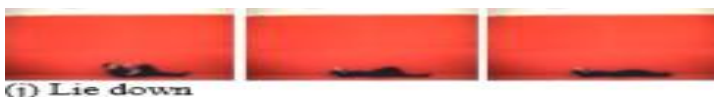

(e) Lying down

\section{Figure3: Normal Activity Behavior}

b. Unusual (Falling): Since most falls occur during intentional movements initiated by the person, they happen mainly forward or backward: stumbling on an obstacle while walking or a backward slip on wet ground. However, in some cases the fall occurs sideways, perhaps during a badly controlled sit-to-stand transfer. In this case, the person frequently tries to grip the wall, as shown in Figure 4.

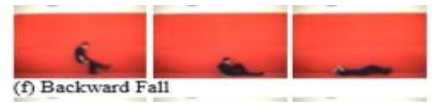

a

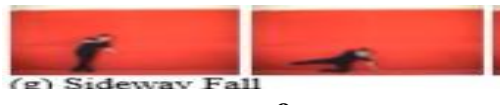

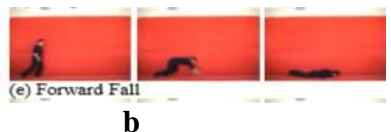

b

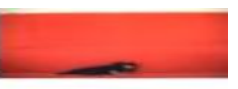

Figure 4: unusual Behavior (a) Forward, (b) Backward,

(c)Sideways

\section{c. Abnormal behavior}

i Stumble: Subjects were asked to walk in an unusual way, e.g. as if they were suffering a balance deficiency such as dizziness. In these situations, a person is unable to maintain balance or symmetry and synchrony of movement. Body movements suggest the person is in a dubious condition, as shown in Figure 5.

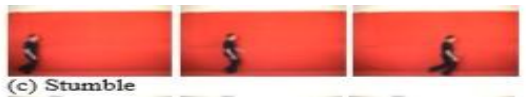

Figure5: Abnormal Behavior (Stumble)

ii Limp: Limping may be caused by unequal leg lengths, experiencing pain when walking, muscle weakness, disorders of proprioception, or stiffness of joints, as shown in Figure6.

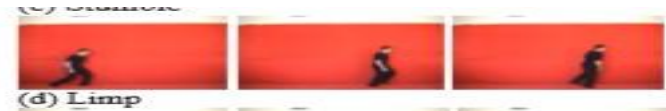

Figure6: Abnormal Behavior (Limp)

Seventy subjects with varying heights, weights, and genders whose ages ranged from 18 to 50 were asked to participate in the project. We repeated 10 kinds of activities 15 times in the experimental space.

\subsection{Evaluation of the system}

Table 1: Recognition rate of different activities

\begin{tabular}{|l|l|l|l|l|}
\hline Events & $\mathbf{A}_{\mathbf{e}}$ & $\mathbf{C}_{\mathbf{e}}$ & $\mathbf{F}_{\mathbf{e}}$ & $\mathbf{R}$ \\
\hline Walk & 80 & 73 & 7 & 91.25 \\
\hline Run & 80 & 75 & 5 & 93.75 \\
\hline Stumble & 80 & 70 & 10 & 87.5 \\
\hline Limp & 80 & 66 & 14 & 82.25 \\
\hline Forward fall & 80 & 70 & 10 & 87.5 \\
\hline Backward fall & 80 & 69 & 11 & 86.25 \\
\hline Sideway fall & 80 & 73 & 7 & 91.25 \\
\hline Bend down & 80 & 75 & 5 & 93.75 \\
\hline Site down & 80 & 71 & 9 & 88.75 \\
\hline Lie down & 80 & 72 & 8 & 90.0 \\
\hline
\end{tabular}

The experimental results show that the system achieved promising results under most test conditions. Table 1shows the recognition rate $(\mathrm{R})$ of the experimental results. (Ae) refers to the number of actions, $(\mathrm{Ce})$ is number of correctly detected events, $(\mathrm{Fe})$ is the number of falsely detected events. To evaluate the system performance, we use two famous criteria that are widely used in fall detection systems. Sensitivity is the capacity to detect a fall, and specificity is the capacity to detect only a fall:

\section{Xpos}

$$
\begin{gathered}
\text { Sensitivity }=\text { Xpos }+ \text { Yneg } \\
\text { Xneg } \\
\text { Specificity }=\text {....... (2) (3) } \\
\text { Xneg +Ypos }
\end{gathered}
$$

The system dataset is composed of video sequences representing 730 normal/abnormal activities and 240 simulated falls. Table 2 itemizes all the results obtained with our dataset. According to this information, the proposed system sensitivity and specificity would be 90.41 and 96.48 ,respectively. 
Table 2: Result of Evolution

\begin{tabular}{|l|l|l|}
\hline $\begin{array}{c}\text { Fall } \\
\text { System } \\
\text { occurrence }\end{array}$ & occurs & Not occur \\
\hline Positive & & \\
\hline Negative & 217 & 26 \\
\hline
\end{tabular}

\section{CONCLUSION}

In this paper, we developed a novel real-time video security system dedicated to behavior detection with a low false alarm rate for human detection and tracking in video sequences and improving response time by designing system that takes timevarying data from multiple cameras to obtain point correspondence and perform calibration. The system tracks a moving object in the scene and uses its location at every time step as a single point. Correspondence among multiple cameras and the architecture enables a single operator to monitor activities over a broad area using a distributed network of video sensors.

\section{REFERENCES}

[1] F.Nilsson ,"Intelligent network video ", CRC Press Taylor \&Francis Group,2009.

[2] H.Qian, X.Wu, and Y.Xu, "Intelligent surveillance systems", Springer,2011.

[3]S.A. Velastin and P.Remagnion, "Intelligent distributed video surveillances systems ",MPG Books,2008.

[4] K.Lee,M.Nam,K. Chung,Y.Lee, and U. Kang, "Context and profile-based cascade classifier for efficient people detection and safetycare system", Springer Science and Business Media, 2012.

[5] M. Betke et al., Tracking Large Variable Numbers of Objects in Clutter ,IEEECVPR, 2007.

[6]P. Antonakaki, D. I. Kosmopoulos,and S. J. Perantonis,“ Detecting abnormal human behavior using multiple cameras," Signal Processing,2009.

[7] J.Zhang, L.Shao, L.Zhang, and G. A. Johns, "Intelligent video, event analysis and Understanding”, Springer,2011.

[8] R.Gumzej, W. A. Haling, "Real-time system quality of service", Springer,2010.

[9] M.Schumacher, "Objective coordination in multi-agent system engineering”, Springer, 2010.

[10] MSarfraz, "Intelligent recognition techniques and application”, John Wiley and Sons,2008.

\section{AUTHOR'S PROFILE}

Abeer Salim Jamil received B.Sc. in Computer Science from Technology University in 1997 and M.Sc. from Computer Science from Technology University in 2004 and $\mathrm{PhD}$ student (research level) in Computer Science from Technology University. Currently she is a Lecture in Computer Science \& Information Systems Department/ AlMansour University College (MUC).Her research interests include Computer vision, Artificial Intelligent, Behavior Analysis , Multi - Agent System Design, Software Engineering, Multi Camera Track and Security.

Abdul Monem S. Rahma received M.Sc. from Brunel University and $\mathrm{PhD}$ from Loughborough University of technology, UK in 1982, 1984 respectively.

Currently, he fills the position of Dean and works as a professor at Computer Science Department, University of Technology, Iraq. He supervised $28 \mathrm{PhD}$ and $57 \mathrm{MSc}$ students. His research interests include image processing, biometrics, computer security, and graphics. 